\title{
The Effect of Single Dose Methylphenidate on Neurometabolites according to COMT Gene Val158Met Polymorphism in the Patient with Attention Deficit Hyperactivity Disorder: A Study Using Magnetic Resonance Spectroscopy
}

\author{
Onder Ozturk ${ }^{1}$, Huseyin Alacam², Burge Kabukcu Basay ${ }^{1}$, Omer Basay ${ }^{1}$, Ahmet Buber ${ }^{1}$, Ozlem Izci Ay ${ }^{3}$, \\ Kadir Agladıoglu', Mehmet Emin Erdal ${ }^{3, *}$, Hasan Herken ${ }^{2, *}$ \\ 'Department of Child and Adolescent Psychiatry, ${ }^{2}$ Department of Psychiatry, ${ }^{4}$ Department of Radiology, Medical Faculty, Pamukkale \\ University, Denizli, ${ }^{3}$ Department of Medical Biology and Genetics, Medical Faculty, Mersin University, Mersin, Turkey
}

\begin{abstract}
Objective: Attention deficit/hyperactivity disorder (ADHD) is a common neurodevelopmental disorder. Thus, the present study aimed to determine the effects of a single dose of methylphenidate (Mph) on neurometabolite levels according to polymorphisms of the catechol-O-methyltransferase (COMT) gene.

Methods: This study evaluated the neurometabolite levels including N-acetylaspartate (NAA), creatine (Cr), and choline (Cho) of $\mathrm{ADHD}$ patients, before and after treatment with $\mathrm{Mph}(10 \mathrm{mg})$ according to the presence of COMT polymorphisms. The spectra were obtained from the dorsolateral prefrontal cortex (DLPFC), anterior cingulate cortex (ACC), cerebellum, and striatum. Results: The NAA levels of the val/val and val genotype carriers (val/val and val/met genotypes) increased in the DLPFC and ACC, respectively, following Mph treatment. The NAA/Cr ratio was lower in the DLPFC of val carriers than in the met/met genotype carriers prior to Mph administration. The Cho levels of the val/met genotype and val carriers increased in the striatum following Mph treatment. Following Mph treatment, the $\mathrm{Cr}$ levels of the met/met genotype carriers were higher than those of the val/met genotype and val carriers. Additionally, after Mph treatment, there was a significant increase in $\mathrm{Cr}$ levels in the DLPFC of the met/met genotype carriers but a significant decrease in such levels in the striatum of val/val genotype carriers.

Conclusion: These findings suggest that polymorphisms of the COMT gene can account for individual differences in neurochemical responses to Mph among ADHD patients. Therefore, further studies are needed to fully characterize the effects of the Val158met polymorphism of the COMT gene on treatment outcomes in patients with ADHD.
\end{abstract}

KEY WORDS: Catechol-O-methyltransferase; Neurometabolite; Attention deficit disorder with hyperactivity; Methylphenidate.

\section{INTRODUCTION}

Attention deficit hyperactivity disorder (ADHD) is a neurodevelopmental disorder that manifests during childhood and is characterized by attentional problems and/or hyperactivity-impulsivity in varying degrees of severity; it has a worldwide pooled prevalence of $5.29 \%{ }^{1)}$ ADHD is a chronic disorder that negatively affects several dimensions of life, particularly the academic, occupational, and social domains, during both childhood and adul-

\footnotetext{
Received: September 13, 2015/ Revised: November 7, 2015

Accepted: December 8, 2015

Address for correspondence: Hasan Herken, MD

Department of Psychiatry, Medical Faculty, Pamukkale University School of Medicine, Camlaraltı neighborhood, No: 4 Kınıklı Campus/ Denizli 20070, Turkey

Tel: +90-532-5540260, Fax: +90-258-296-6001

E-mail: hherken@pau.edu.tr

*These authors contributed equally to this study.
}

thood. ${ }^{2)}$ In terms of its etiology, there are disruptions of the dopaminergic and noradrenergic pathways, which regulate attention, and an inhibition of executive function via alterations of the cortico- $^{-}$striato-thalamic- $^{-}$cortical net- $^{-}$ works. $^{3,4)}$

The genetic heritability of ADHD ranges from $70 \%$ to $90 \%,{ }^{2,5,6)}$ and a number of studies have investigated candidate genes that may contribute to the manifestation of ADHD. The catechol- $O$-methyltransferase (COMT) gene, which plays a role in the removal of dopamine (DA) from the synaptic space, is frequently studied as a possible candidate gene. ${ }^{7}$ A single nucleotide polymorphism (SNP) of the COMT gene that includes a guanine $(\mathrm{G})$ to adenine (A) mutation at codon 158 results in an amino acid substitution of methionine (met) for valine (val) during enzyme synthesis. ${ }^{8)}$ This polymorphism, which is known as either Val158Met or rs $4680,{ }^{8)}$ results in an enzyme with two Val alleles (val/val genotype) that is more thermo-

(c) This is an Open-Access article distributed under the terms of the Creative Commons Attribution Non-Commercial License (http://creativecommons.org/licenses/by-nc/4.0) which permits unrestricted non-commercial use, distribution, and reproduction in any medium, provided the original work is properly cited 
stable $^{9)}$ and degrades DA three-to-four times more rapidly than the two met isoforms (met/met genotypes) which, in turn, results in lower levels of DA in the synaptic space. ${ }^{8)}$

Heterozygotes (val/met genotype carriers) are associated with intermediate levels of COMT activity, ${ }^{10,11)}$ and healthy met/met genotype carriers exhibit superior performance in a number of cognitive paradigms, including the letter-number-sequencing test ${ }^{12)}$ and n-back task, ${ }^{13)}$ compared with individuals with the val/val genotype. The Val allele is related to decreased prefrontal cortical activation in healthy adults ${ }^{14)}$ and impaired working memory function in healthy adults and children. ${ }^{14,15)}$ Cheon et al. ${ }^{16)}$ evaluated the association between the Val158met polymorphism and treatment response and found that the response to methylphenidate (Mph) was better in val/val genotype carriers than in carriers of other genotypes. However, Bellgrove et al. ${ }^{17)}$ observed that children with ADHD carrying the val/val genotype exhibited superior sustained attention than met carriers (met/met and val/met genotypes). On the other hand, studies of the relationship between the etiology of ADHD and polymorphisms of the COMT gene have reported equivocal results. For example, Kereszturi et al ${ }^{18)}$ reported that the Val allele is more common in children with ADHD than in healthy controls, whereas other studies ${ }^{19-21)}$ and meta-analyses ${ }^{22,23)}$ did not observe any type of relationship between these variables.

${ }^{1} \mathrm{H}$ magnetic resonance spectroscopy $\left({ }^{1} \mathrm{H} \mathrm{MRS}\right)$ is a non-invasive neuroimaging technique that can be used in vivo to assess levels of neurometabolites, such as $\mathrm{N}$-acetylaspartate (NAA), creatine $(\mathrm{Cr})$, and choline (Cho), in a variety of brain areas. Courvoisie et al. ${ }^{24)}$ found higher levels of Cho in the prefrontal cortex (PFC) of children diagnosed with ADHD compared with healthy controls. Similarly, Husarova et $a l^{25}{ }^{25}$ assessed children with ADHD during the second month of atomoxetine treatment and identified decreases in NAA levels and the NAA/Cr ratio in the left dorsolateral PFC (DLPFC) in conjunction with an increase in the $\mathrm{Cho} / \mathrm{Cr}$ ratio in the right DLPFC. Amor $^{26)}$ reported that drug-naïve children with a diagnosis of ADHD had lower Cho levels in the left PFC compared with healthy controls and ADHD children who had received treatment. A meta-analysis conducted by Aoki et $a l .{ }^{27)}$ showed that, although the results of the adult ADHD patients and controls were similar, the NAA levels of ADHD-diagnosed children were higher than those of healthy controls in the medial PFC area. Thus, the present authors speculated that age-related changes in ADHD symptoms may have a neuronal basis even though no significant alterations in other metabolites have been reported.

Therefore, the present study utilized ${ }^{1} \mathrm{H}$ MRS to investigate alterations in the neurometabolite levels of adult ADHD patients following a single dose of Mph according to the presence of the Val158met polymorphism of the COMT gene. To the best of our knowledge, the present study is the first to investigate pre- and post-Mph neurometabolite levels in adult ADHD patients based on this COMT polymorphism. It was hypothesized that different COMT gene polymorphism carriers would exhibit different treatment outcomes in terms of neurometabolite levels in different brain regions following a single dose of $\mathrm{Mph}$.

\section{METHODS}

\section{Subject Characteristics}

The present study assessed 57 adult patients who were between 18 and 60 years of age and who met the criteria of the Diagnostic and Statistical Manual of Mental Disorders 4th edition, text revision (DSM-IV-TR) for ADHD. ${ }^{28)}$ All patients were recruited from the psychiatry outpatient clinic of Pamukkale University in Turkey, and the absence of other psychiatric diagnoses was confirmed by structured interviews using the Turkish version of the Structured Clinical Interview for DSM-IV Clinical Version (SCID-I CV). ${ }^{29)}$ Additionally, the Turkish version of an adult attention-deficit disorder (ADD)/ADHD DSM-IVbased diagnostic screening and rating scale was used to assess the patients. ${ }^{30)}$ Patients with a neurodegenerative disorder, such as Alzheimer's disease or Parkinson's disease, and/or a clinical evaluation of an intellectual disability were excluded from the present study. The local Ethics Committee of Pamukkale University approved the study protocol (approval no. 60116787/020/27537), and all patients were informed of the aim of the study and its procedures; all patients also provided written informed consent confirming their voluntary participation in this study.

All patients were examined with a single-voxel ${ }^{1} \mathrm{H}$ MRS, and the spectra were obtained from the DLPFC, anterior cingulate cortex (ACC), striatum, and cerebellum via a clinical 1.5-T magnetic resonance scanner (GE Medical System, Milwaukee, WI, USA). For this procedure, the following parameters were employed: a point-resolved spectroscopy sequence with water suppression, a chemical shift selective imaging sequence, an echo time (TE)/repetition time (TR) ratio of 35/3,000 ms, and 128 averages. Additionally, the present study utilized a conventional spin-echo sequence with T2-weighted fast spin-echo parameters as follows: horizontal slices (10-mm 
thick), a TR/TE ratio of 3,000/88 ms, a field of view of 10 $\mathrm{cm}$, and a $512 \times 512$ matrix. The entire MR session took approximately 45 minutes to complete.

Patients who had been previously been administered Mph discontinued their use of the medication 48 hours prior to the initiation of the neuroimaging procedures. The levels of NAA, Cho, and Cr in the DLPFC, ACC, striatum, and cerebellum were examined using single-voxel ${ }^{1} \mathrm{H}$ MRS when the patients were medication-naïve $30 \mathrm{mi}$ nutes after the administration of $\mathrm{Mph}(10 \mathrm{mg})$.

\section{Genotypic Analysis of the Val158Met (c.1947, G>A, rs4680) Polymorphism of the COMT Gene}

\section{DNA extraction}

A venous blood sample was obtained from each individual and collected in a tube containing ethylenediaminetetraacetic acid. The DNA was extracted from the whole blood using a previously described salting-out procedure. $^{31)}$

\section{Genotype determination}

The genotypes were determined using a TaqMan ${ }^{\mathrm{TM}}$ fluorogenic 5'-nuclease assay and TaqMan probes (Applied Biosystems, Framingham, MA, USA). The Primer Express 3.0 software package (Applied Biosystems) was used to design both the polymerase chain reaction (PCR) primers and the TaqMan probes. The following custommade primers and probes were used for the COMT gene 1947 G > A Val158Met (rs4680) allele; forward primer: 5'-CATCACCCAGCGGATGGT-3', reverse primer: 5'TCAGGCATGCACACCTTGTC-3', probe: (A)5'-VICATTTCGCTGGCATGAA-NFQ-MGB-3', and probe: (G) 5'-FAM-ATTTCGCTGGCGTGAA-NFQ-MGB-3' (Applied Biosystems).

The SNP amplification assays were performed according to the manufacturer's instructions (Applied Biosystems). Briefly, $25 \mu 1$ of the reaction solution that contained $30 \mathrm{ng}$ of DNA was mixed with $12.5 \mu 1$ of $2 \times$ TaqMan Universal PCR Master Mix (Applied Biosystems), $900 \mathrm{nmol}$ of each primer, and $200 \mathrm{nmol}$ of each probe. The reaction conditions were as follows: pre-incubation at $60^{\circ} \mathrm{C}$ for 1 minute, incubation at $95^{\circ} \mathrm{C}$ for 10 minutes, 40 cycles at $95^{\circ} \mathrm{C}$ for 15 seconds, and then incubation at $60^{\circ} \mathrm{C}$ for 1 minute. The amplifications and analyses were performed using an ABI Prism 7500 Real-Time PCR System (Applied Biosystems) with the SDS software package (ver. 2.0.6; Applied Biosystems) for allelic discrimination. ${ }^{31)}$

\section{Statistical Analysis}

All data were analyzed using the SPSS for Windows software package (ver. 17.0; SPSS Inc., Chicago, IL, USA). Categorical variables were compared using Pearson's chi-square $\left(\chi^{2}\right)$ tests, and the distribution of the numeric variables was evaluated using the KolmogorovSmirnov test. Nonparametric tests were used because the variables were not distributed normally. Kruskal-Wallis tests were used to make comparisons among the three groups, and Mann-Whitney $U$-tests were used to compare the data between two groups. Significant differences in the changes in neurometabolite levels prior to and after treatment with $\mathrm{Mph}$ were assessed with the Wilcoxon two-related-samples test, and a $p$ value $<0.05$ was considered to indicate statistical significance.

\section{RESULTS}

Of the 57 adult ADHD patients assessed in the present study, 12 were female (21.1\%) and 45 were male (78.9\%); all were between 18 and 60 years of age (mean age, $29.05 \pm 7.85$ years). However, only $33(57.9 \%)$ of the patients had been receiving Mph for the treatment of ADHD (Table 1).

The COMT polymorphism and ADHD subtype distributions of the patients are shown in Table 2. The distribution of the three genotypes for the COMT gene was in agreement with the expected values of the HardyWeinberg equilibrium $(p=0.888)$. The majority of the patient group $(80.7 \% ; \mathrm{n}=46)$ were val carriers ( $\mathrm{val} / \mathrm{val}$, $\mathrm{val} / \mathrm{met}$ ), that is, rapid metabolizers. There were no significant differences in the distributions of the ADHD sub-

Table 1. Sociodemographic characteristics

\begin{tabular}{lc}
\hline Characteristic & Data \\
\hline Sex & $12(21.1)$ \\
Female & $45(78.9)$ \\
Male & \\
Marital status & $35(61.4)$ \\
Single & $18(31.6)$ \\
Married & $4(7.0)$ \\
Divorced & \\
Education & $5(8.8)$ \\
Primary education & $12(21.1)$ \\
High school & $40(70.1)$ \\
University & \\
Methylphenidate use & $33(57.9)$ \\
Yes & $24(42.1)$ \\
No &
\end{tabular}

Values are presented as number (\%). 
types according to COMT polymorphism $\left(\chi^{2}=4.29\right.$, $p=0.29$; Table 2). In total, 37 of the males (82.2\%) and nine of the females $(75.0 \%)$ were val carriers, but there was no significant difference in the gender distribution according to COMT polymorphism $\left(\chi^{2}=0.31, p=0.57\right)$.

For the metabolic assessments, the patients were categorized according to the COMT polymorphisms in two different ways, and all statistical analyses were performed accordingly. For the first categorization, the patients were divided into three groups (val/val, val/met, and met/met

Table 2. ADHD subtypes and the COMT gene polymorphism

\begin{tabular}{|c|c|c|c|}
\hline & \multicolumn{3}{|c|}{ COMT gene polymorphism } \\
\hline & Val/val & Val/met & Met/met \\
\hline Attention deficit type & $9(45.0)$ & $8(40.0)$ & $3(15.0)$ \\
\hline Hyperactivity impulsivity type & $5(55.6)$ & $3(33.3)$ & $1(11.1)$ \\
\hline Combined type & $6(21.4)$ & $15(53.6)$ & $7(25.0)$ \\
\hline Total & $20(35.1)$ & $26(45.6)$ & $11(19.3)$ \\
\hline
\end{tabular}

Values are presented as number (\%).

ADHD, attention deficit hyperactivity disorder; COMT, catechol-Omethyltransferase.

$p=0.29, X^{2}=4.29$; by chi-square test. genotype carriers) and their neurometabolic variables were compared. For the second categorization, the patients were divided into two groups: val genotype carriers (val/val and val/met; i.e., rapid metabolizers) and met/met genotype carriers (slow metabolizers). After a single 10-mg dose of $\mathrm{Mph}$, there was a significant increase in NAA levels in the DLPFC of the val/val genotype carriers $(p=0.04)$ and the ACC of the val carriers $(p=0.03$; Table 3 and Fig. 1). The pre-Mph NAA/Cr ratio was significantly lower in the DLPFC of val carriers than that in the met/met genotype carriers $(p=0.02)$, but there were no significant post-Mph alterations in NAA levels or NAA/Cr ratios in the other brain regions that were investigated. Cho levels in the striatum of the val/met and val genotype carriers significantly increased after a single dose of Mph compared with pre-medication levels $(p=0.01$ and $p=0.007$, respectively). However, there were no significant medication-related changes in Cho levels in the other brain regions based on polymorphism (Table 3 and Fig. 1). Although the pre-medication Cr levels in the DLPFC were similar in all groups, after Mph treatment there were higher $\mathrm{Cr}$ levels in the met/met genotype carriers than in the

Table 3. Comparison of the neurometabolites between the COMT gene Val158Met polymorphism groups

\begin{tabular}{|c|c|c|c|c|c|c|}
\hline & & Val/val & Val/met & Met/met & $p$ value* & $p$ value $^{\dagger}$ \\
\hline \multicolumn{7}{|c|}{ Comparison of NAA levels before and after Mph } \\
\hline \multirow[t]{2}{*}{ DLPFC } & Before Mph & $68(50-117)^{\dagger}$ & $66(45-98)$ & $74(53-107)$ & 0.486 & $0.04^{\dagger}$ \\
\hline & After Mph & $71(51-115)^{\dagger}$ & $59.5(39-92)$ & $70(60-113)$ & 0.097 & \\
\hline \multirow[t]{2}{*}{$\mathrm{ACC}$} & Before Mph & $62.5(51-126)$ & $58(39-87)$ & $66(51-83)$ & 0.174 & \\
\hline & After Mph & $68.5(54-113)$ & $63(47-94)$ & $62(56-79)$ & 0.225 & \\
\hline \multirow[t]{2}{*}{ Striatum } & Before Mph & $63.5(50-97)$ & $62(45-82)$ & $61(52-76)$ & 0.684 & \\
\hline & After Mph & $67(45-94)$ & $59(45-85)$ & $68(55-105)$ & 0.146 & \\
\hline \multirow[t]{2}{*}{ Cerebellum } & Before Mph & $61(38-96)$ & $60.5(31-78)$ & $57(37-89)$ & 0.724 & \\
\hline & After Mph & $66(38-85)$ & $60.5(38-84)$ & $61(52-88)$ & 0.521 & \\
\hline \multicolumn{7}{|c|}{ Comparison of Cho levels before and after Mph } \\
\hline \multirow[t]{2}{*}{ DLPFC } & Before Mph & $41(26-66)$ & $38(29-62)$ & $37(26-52)$ & 0.196 & \\
\hline & After Mph & $42(29-70)$ & $39.5(26-62)$ & $40(36-51)$ & 0.170 & \\
\hline \multirow[t]{2}{*}{ ACC } & Before Mph & $38.5(24-65)$ & $37(25-50)$ & $38(22-54)$ & 0.945 & \\
\hline & After Mph & $41(24-44)$ & $37.5(26-52)$ & $35(32-48)$ & 0.405 & \\
\hline \multirow[t]{2}{*}{ Striatum } & Before Mph & $36(24-53)$ & $33(22-45)^{\dagger}$ & $36(28-55)$ & 0.239 & $0.01^{\dagger}$ \\
\hline & After Mph & $36.5(27-57)$ & $35.5(27-56)^{\dagger}$ & $36(26-47)$ & 0.929 & \\
\hline \multirow[t]{2}{*}{ Cerebellum } & Before Mph & $45.5(31-61)$ & $40.5(25-51)$ & $45(25-59)$ & 0.196 & \\
\hline & After Mph & $41(27-64)$ & $41.5(29-56)$ & $44(31-64)$ & 0.492 & \\
\hline \multicolumn{7}{|c|}{ Comparison of $\mathrm{Cr}$ levels before and after Mph } \\
\hline \multirow[t]{2}{*}{ DLPFC } & Before Mph & $40(29-70)$ & $40.5(29-53)$ & $39(28-55)^{\dagger}$ & 0.804 & $0.01^{\dagger}$ \\
\hline & After Mph & $43(33-69)$ & $39(23-60)^{*}$ & $42(38-66)^{*}+$ & $0.018^{*}$ & \\
\hline \multirow[t]{2}{*}{ ACC } & Before Mph & $41.5(29-73)$ & $40.5(30-57)$ & $43(36-49)$ & 0.357 & \\
\hline & After Mph & $44(31-69)$ & $42.5(33-62)$ & $43(34-55)$ & 0.591 & \\
\hline \multirow[t]{2}{*}{ Striatum } & Before Mph & $47.5(36-69)^{\dagger}$ & $42(29-51)$ & $46(38-59)$ & 0.054 & $0.01^{\dagger}$ \\
\hline & After Mph & $43(34-66)^{\dagger}$ & $41.5(34-58)$ & $48(36-61)$ & 0.463 & \\
\hline \multirow[t]{2}{*}{ Cerebellum } & Before Mph & $53(24-70)$ & $49(34-66)$ & $54(29-65)$ & 0.396 & \\
\hline & After Mph & $52(27-74)$ & $52.5(27-65)$ & $55(41-74)$ & 0.542 & \\
\hline
\end{tabular}

Values are presented as median (range)

COMT, catechol-O-methyltransferase; DLPFC, dorsolateral prefrontal cortex; ACC, anterior cingulate cortex; Mph, methylphenidate; NAA, $\mathrm{N}$-acetylaspartate; Cho, choline; Cr, creatine. 


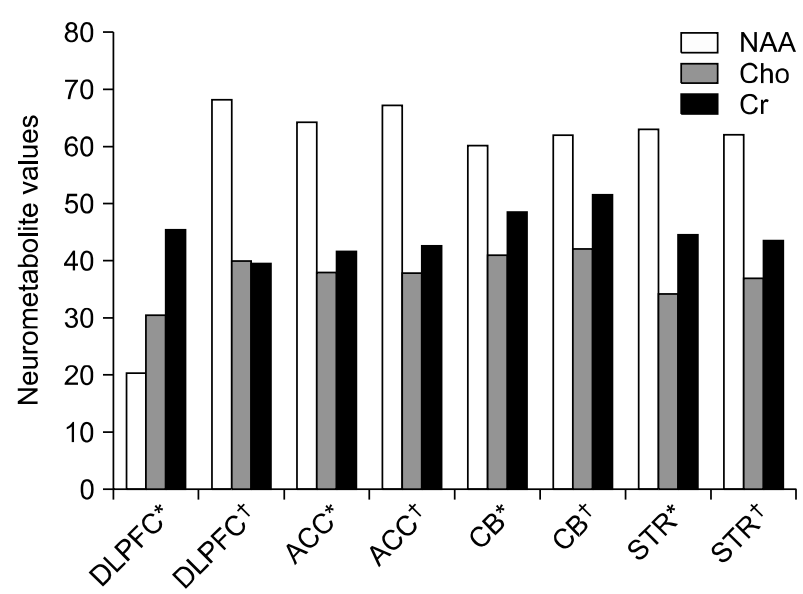

Fig. 1. Values of neurometabolites before and after methylphenidate in Val carriers (rapid metabolizers).

DLPFC, dorsolateral prefrontal cortex; ACC, anterior cingulate cortex; CB, cerebellum; STR, striatum; NAA, N-acetylaspartate; Cho, choline; $\mathrm{Cr}$, creatinine.

*Before methylphenidate, ${ }^{\dagger}$ after methylphenidate.

$\mathrm{val} / \mathrm{met}$ and val carriers $(p=0.01$ and $p=0.03$, respectively; Table 3). Consistent with these findings, the $\mathrm{Cr}$ levels in the DLPFC of the met/met genotype carriers significantly increased after a single dose of Mph compared with their pre-medication levels ( $p=0.01$; Table 3 and Fig. 2 ). In the $\mathrm{val} / \mathrm{val}$ genotype carriers, $\mathrm{Cr}$ levels in the striatum significantly decreased after Mph treatment $(p=0.01)$, but the $\mathrm{Cr}$ levels in the other brain regions were not affected (Table 3).

\section{DISCUSSION}

Of the 57 adult ADHD patients assessed in the present study, the number of rapid metabolizers (val carriers; $\mathrm{n}=46$ ) was approximately fourfold higher than that of slow metabolizers (met/met genotype; $n=11$ ). The Val allele enhances the hypo-dopaminergic state in the synaptic space to a greater degree than the Met allele due to its thermostability, ${ }^{9)}$ and healthy met/met genotype carriers exhibit superior cognitive performance compared with val/val genotype carriers. ${ }^{12,13)}$ Similarly, the Val allele has a stronger association with impaired working memory function than does the Met allele in healthy adults and children, ${ }^{14,15)}$ and it is also associated with decreased prefrontal cortical activation in healthy adults. ${ }^{14)}$ In contrast to these findings, other studies ${ }^{24,32)}$ and meta-analyses ${ }^{22,23)}$ have reported no relationship between ADHD and COMT gene polymorphisms.

The predominance of val carriers in the present patient group appears to support the Val allele-ADHD relation-

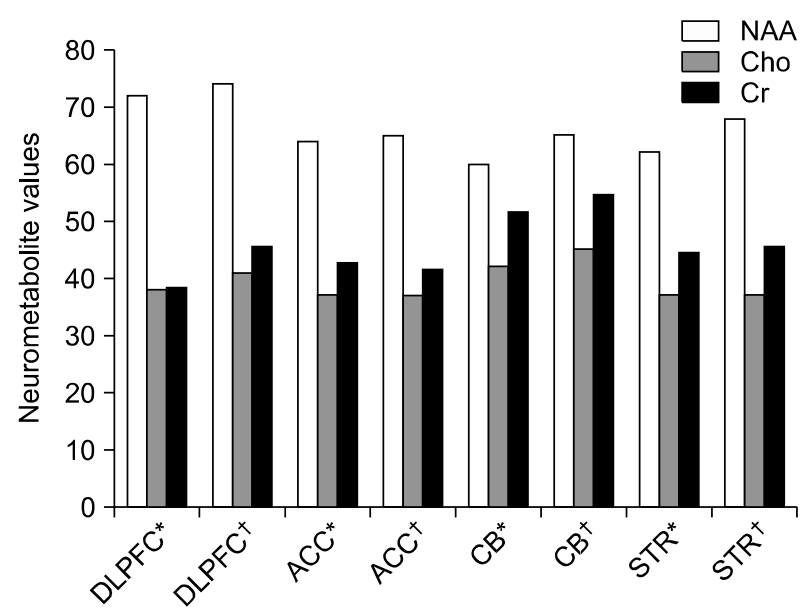

Fig. 2. Values of neurometabolites before and after methylphenidate in met/met genotypes (slow metabolizers).

DLPFC, dorsolateral prefrontal cortex; ACC, anterior cingulate cortex; $C B$, cerebellum; STR, striatum; NAA, N-acetylaspartate; Cho, choline; $\mathrm{Cr}$, creatinine.

*Before methylphenidate, ${ }^{\dagger}$ after methylphenidate.

ship in terms of disease etiology. The conflicting reports from several candidate gene studies may be due to the multigenic and multifactorial etiology of ADHD because, although the COMT polymorphism may affect the development of ADHD, this does not occur in isolation. To better understand this relationship, ADHD-related networks and other factors (particularly catecholamine-related polymorphisms) that may have an impact on the development of ADHD should be researched on a larger scale. Consistent with the results of Yatsuga et al., ${ }^{32)}$ the present study did not find a relationship between ADHD subtype and COMT polymorphism. It is possible that the contribution of the COMT polymorphism to ADHD is not subtype-specific or it could be that the small sample size used in the present study may have influenced the present results.

\section{NAA}

Following treatment with $\mathrm{Mph}$, there was a significant increase in the amount of NAA in the DLPFC of the $\mathrm{val} / \mathrm{val}$ genotype carriers and in the $\mathrm{ACC}$ of the val carriers. In contrast, Carrey et al. ${ }^{33)}$ did not find any significant alterations in the neurometabolite levels in the right frontal region of ADHD patients after 13 weeks of treatment with atomoxetine, Mph, or Dexedrine. Husarova et al. ${ }^{25)}$ reported that the NAA levels and NAA/Cr ratio decreased in the left DLPFC of ADHD patients after 2 months of atomoxetine treatment, but that there were no significant alterations after treatment with Mph. A metaanalysis revealed that the amount of NAA in the medial 
PFC of children with ADHD was significantly higher than that of the controls, but this difference declined with age and disappeared in adults, which suggests that age-related alterations in ADHD symptoms may have a neuronal basis. $^{34)}$

In this respect, the present findings disagree with those of previous studies. NAA is considered to be a marker of regional neuronal activation and vitality, ${ }^{35}$ and neuroimaging studies conducted among ADHD patients have observed numerous alterations of this metabolite in various brain regions, principally the PFC, ACC, and striatum. ${ }^{36)}$ Compared with healthy controls, ADHD patients show reduced perfusion, especially in the $\mathrm{PFC},{ }^{37,38)}$ and a study investigating the effects of treatment on hypoperfusion found that stimulant medication increases blood flow in the bilateral prefrontal, caudate, and thalamic areas. ${ }^{38)}$ Similarly, the chronic administration of Mph increases neuronal mitochondrial activity in rats, ${ }^{39)}$ and a single dose of Mph can lead to increases in neuronal activation in the frontal lobes of patients with ADHD. ${ }^{40)}$

Taken together, these data suggest that Mph has a positive impact on impaired cerebral perfusion and neuronal function. Therefore, post-Mph increases in NAA levels may indicate improved perfusion and increased neuronal activation, as in the present data. As suggested by Angelie et al. ${ }^{41)}$ the contrasting results among studies may be due to the presence or absence of a cognitive performance measure during the assessment process and, more importantly, they may be related to age, sex, the investigated regions, or related variations in NAA levels.

\section{$\mathrm{NAA} / \mathrm{Cr}$ Ratio}

In the present study, the pre-Mph NAA/Cr ratio in the DLPFC of val carriers was significantly lower than that of the met/met genotype carriers. During the literature search for this study, it became clear that no previous studies had investigated the relationship between the $\mathrm{NAA} / \mathrm{Cr}$ ratio and COMT polymorphisms. Jin et $\mathrm{al}^{42)}$ reported a lower $\mathrm{NAA} / \mathrm{Cr}$ ratio in the striatum of children with ADHD relative to that of healthy controls, and this did not change after a single 10-mg dose of Mph. In contrast, Fayed et al. ${ }^{43)}$ found that the prefrontal cortico-subcortical NAA/Cr ratio is higher in ADHD children than in healthy controls, and Wiguna et $\mathrm{al}^{44)}$ reported a significantly increase in the $\mathrm{NAA} / \mathrm{Cr}$ ratio in the bilateral PFC of ADHD patients after 12 weeks of Mph treatment. Contradictory results in the literature may be due to changes in NAA levels according to age, sex, and the brain area investigated.

The NAA/Cr ratio is a marker of brain maturation. ${ }^{45)}$
Childhood ADHD is related to delayed cortical maturation, especially in the PFC. ${ }^{4)}$ Furthermore, adult studies have demonstrated the presence of decreased gray matter and reduced cortical thickness in ADHD patients compared with controls. ${ }^{46,47)}$ It is known that cortical thickness is related to disease severity. ${ }^{47)}$ The number of Val alleles in ADHD patients is associated with the rate at which DA is metabolized ${ }^{9-11)}$ and, therefore, it is also related to disease severity. This indicates that the present finding that val carriers have a lower NAA/Cr ratio (a maturation marker) than the met/met genotype carriers is intuitive.

\section{Cho}

Cho levels significantly increased in the striatum of the val and val/met genotype carriers following treatment with Mph compared with pre-medication levels. To the best of our knowledge, no studies have investigated the relationship between Cho levels in ADHD patients and COMT polymorphisms. A number of studies have assessed Cho metabolites in ADHD patients and produced variable results. Similar to the present findings, Carrey et $a l{ }^{33)}$ did not find any treatment-related changes in Cho levels in the PFC of ADHD children. However, this negative result in the striatal area contrasts with the findings of the present study. Kronenberg et al. ${ }^{48)}$ found a significant decrease in the Cho levels in the ACC of adult ADHD patients after 5-6 weeks of Mph treatment. In a study conducted by Amor, ${ }^{26)}$ lower levels of Cho were reported in the left prefrontal area of treatment-naïve ADHD patients compared with both treatment-receiving ADHD children and healthy controls. Jin et al. ${ }^{42)}$ reported a marginal increase in the $\mathrm{Cho} / \mathrm{Cr}$ ratio in the striatum of children with ADHD, but a single 10-mg dose of oral Mph had no effect on these ratios. The authors speculated that this could account for approximately $20-25 \%$ of the neural loss and/or dysfunction observed in ADHD patients.

Cho is found in the cellular membrane and is an indicator of lipid metabolism and membrane integrity. ${ }^{49)}$ It should also be noted that Cho is an acetylcholine precursor that influences neural communication and is mediated by various neurotransmitters, including norepinephrine and DA. It has been suggested that impairments in dopaminergic pathways, as well as the imbalances in the dopaminergic-cholinergic system that are seen in ADHD, may affect Cho levels. ${ }^{42)}$ With respect to previous studies, these differences may be related to medication type, treatment duration and dose, and variations in the genetic structure of the recruited individuals. However, $57.9 \%$ of the present patients were medicated with Mph and, there- 
fore, the data may have been influenced by drug-induced effects on neuroplasticity.

\section{Cr}

Following the administration of $\mathrm{Mph}$ in the present study, Cr levels in the DLPFC were significantly higher in the met/met genotype carriers than in the val/met genotype carriers and val carriers. In accordance with this result, $\mathrm{Cr}$ levels significantly increased in the DLPFC of met/met genotype carriers after Mph treatment compared with pre-Mph levels, whereas the striatal Cr levels in $\mathrm{val} / \mathrm{val}$ genotype carriers significantly decreased after Mph treatment.

To the best of our knowledge, the Cr levels of ADHD patients have yet to be assessed according to COMT polymorphisms, and studies investigating variability in preand post-Mph $\mathrm{Cr}$ levels in ADHD patients have reported contrasting results. Yang et al. ${ }^{50)}$ observed lower $\mathrm{Cr}$ and phospho-Cr levels in the right PFC of ADHD patients compared with healthy controls, whereas a meta-analysis revealed no difference in medial $\mathrm{PFC} \mathrm{Cr}$ levels between ADHD and control groups. ${ }^{27)}$ Consistent with the present results, Kronenberg et al. ${ }^{48)}$ did not find any significant differences in pre- and post-treatment $\mathrm{Cr}$ levels in the ACC of adult ADHD patients after 5-6 weeks of Mph administration.

$\mathrm{Cr}$ and phospho- $\mathrm{Cr}$ are accepted indicators of energy metabolism in neurons and astrocytes. Therefore, decreased levels of these parameters indirectly signify impaired neuronal activity and function. ${ }^{51)}$ In the present study, val genotype carriers had lower prefrontal $\mathrm{Cr}$ levels than met/met genotype carriers, which supports the energetic hypothesis of $\mathrm{ADHD}^{49)}$ and is consistent with the val amino acid-associated hypo-dopaminergic state observed in ADHD patients. ${ }^{9)}$ Following Mph treatment, there was a significant increase in Cr levels in the PFC of the met/met genotype carriers, which indicates that there was an increase in neuronal activity following treatment-related improvements in hypoperfusion. In another study, baseline striatal $\mathrm{Cr}$ levels were reportedly higher in ADHD children than in controls after 8 weeks of treatment and, similar to the present findings, striatal $\mathrm{Cr}$ levels significantly decreased. ${ }^{52)}$ The authors speculated that stimulant treatment might increase monoamine availability and striatal Cr within the context of a phospho-Cr-dependent uptake of glutamate into synaptic vesicles. Therefore, decreased post-medication Cr levels in the striatum may represent a compensatory mechanism. ${ }^{52}$

It should be noted that approximately half the partic- ipants in the present study had previously used Mph for the treatment of ADHD. Previous data have shown that chronic stimulant use influences the activities of neurotransmitter systems and brain plasticity, including synaptic and non-synaptic levels. ${ }^{53,54)}$ Despite contradictory findings, ${ }^{40,55)}$ neuroimaging studies have demonstrated that Mph predominantly acts in the frontal cortex, basal ganglia (including the striatum), ACC, and cerebellum in ADHD patients and that psychostimulant medications generally normalize ADHD-related hypoactivation in these areas. ${ }^{54,55)}$ Studies investigating the effects of psychostimulants on the brains of ADHD patients indicate that these medications cause structural and developmental changes in important brain regions, such as the cerebellar vermis, ${ }^{56)} \mathrm{ACC},{ }^{57)}$ and basal ganglia. ${ }^{58)}$ Conversely, this type of medication is not related to the slowed growth of the cortical mantle in ADHD patients. ${ }^{59)}$ Similarly, volume abnormalities in the cerebrum and cerebellum of ADHD patients are consistently identified across the childhood and adolescent periods, and these changes are not associated with medication use. ${ }^{60)}$ In light of these data, it can be suggested that the present findings might have been affected by the previous use of $\mathrm{Mph}$, even though Mph treatment was discontinued 48 hours prior to the neuroimaging to minimize its effect on neurometabolites. This may have prevented the identification of significant findings specifically related to the cerebellum due to the effects of chronic Mph use on the cerebellum. Thus, future studies are needed to evaluate the effects of Mph among ADHD patients who are drug-naïve.

It is also important to mention that neurometabolite levels, particularly NAA, are affected by age ${ }^{27,41)}$ and clinical presentation $^{61)}$ and that the neurobiological basis of ADHD changes with age. ${ }^{27,34)}$ Therefore, the wide age range of the present sample may have affected the present results, which makes generalizing these findings difficult.

There are several limitations to the present study. Approximately half the patients had already used Mph for the treatment of ADHD, and cigarette use, which may change the response to $\mathrm{Mph}$, was not utilized as an exclusion criterion. In the present study, a low Tesla MR scanner was used, and only unilateral brain assessments were conducted. Additionally, the sample size was relatively small, no control group was included, and the wide age range of the patients decreases the generalizability of the results. Finally, although the patients were evaluated using adult ADD/ADHD DSM-IV-based diagnostic screening and rating scales prior to the administration of $\mathrm{Mph}$, there were no assessments of treatment outcomes 
following the administration of the drug.

The present study assessed the effects of single-dose Mph on three different neurometabolites in adult ADHD patients who were categorized according to COMT polymorphism. As expected, there was a predominance of val carriers (rapid metabolizers) among the patients. Pre- and post-medication changes in neurometabolite levels were detected in certain brain areas in accordance with COMT polymorphisms, which suggests that COMT gene polymorphisms can account for individual differences in the neurochemical response to Mph in ADHD patients. The present study provides important contributions to the literature, because it is the first to investigate the effects of single-dose Mph on neurometabolite levels according to COMT polymorphisms.

To better understand the neuropathology of ADHD, further research using drug-naïve patients, larger sample sizes, and higher-resolution neuroimaging methods is required. Additionally, the effects of age and sex should be minimized, control groups should be included, and the specific effects of the COMT val158met polymorphism on treatment outcomes in ADHD patients should be analyzed.

\section{Acknowledgments}

The authors thank Ayse Gonca Unal for her contribution during the patient recruitment phase.

\section{REFERENCES}

1. Polanczyk G, de Lima MS, Horta BL, Biederman J, Rohde LA. The worldwide prevalence of ADHD: a systematic review and metaregression analysis. Am $J$ Psychiatry 2007; 164:942-948.

2. Faraone SV, Perlis RH, Doyle AE, Smoller JW, Goralnick JJ, Holmgren MA, et al. Molecular genetics of attentiondeficit/hyperactivity disorder. Biol Psychiatry 2005;57: 1313-1323.

3. Sonuga-Barke EJ. Causal models of attention-deficit/hyperactivity disorder: from common simple deficits to multiple developmental pathways. Biol Psychiatry 2005;57:12311238.

4. Shaw P, Eckstrand K, Sharp W, Blumenthal J, Lerch JP, Greenstein D, et al. Attention-deficit/hyperactivity disorder is characterized by a delay in cortical maturation. Proc Natl Acad Sci U S A 2007;104:19649-19654.

5. Faraone SV, Sergeant J, Gillberg C, Biederman J. The worldwide prevalence of $A D H D$ : is it an American condition? World Psychiatry 2003;2:104-113.

6. Chang Z, Lichtenstein P, Asherson PJ, Larsson H. Developmental twin study of attention problems: high heritabilities throughout development. JAMA Psychiatry 2013;70:311318 .

7. Axelrod J. O-methylation of epinephrine and other catechols in vitro and in vivo. Science 1957;126:400-401.

8. Lachman HM, Papolos DF, Saito T, Yu YM, Szumlanski
CL, Weinshilboum RM. Human catechol-O-methyltransferase pharmacogenetics: description of a functional polymorphism and its potential application to neuropsychiatric disorders. Pharmacogenetics 1996;6:243-250.

9. Lotta T, Vidgren J, Tilgmann C, Ulmanen I, Melén K, Julkunen I, et al. Kinetics of human soluble and membrane-bound catechol O-methyltransferase: a revised mechanism and description of the thermolabile variant of the enzyme. Biochemistry 1995;34:4202-4210.

10. Chen J, Lipska BK, Halim N, Ma QD, Matsumoto M, Melhem S, et al. Functional analysis of genetic variation in catechol-O-methyltransferase (COMT): effects on $m R N A$, protein, and enzyme activity in postmortem human brain. Am J Hum Genet 2004;75:807-821.

11. Weinshilboum RM, Otterness DM, Szumlanski CL. Methylation pharmacogenetics: catechol O-methyltransferase, thiopurine methyltransferase, and histamine N-methyltransferase. Annu Rev Pharmacol Toxicol 1999;39:19-52.

12. Bruder GE, Keilp JG, Xu H, Shikhman M, Schori E, Gorman JM, et al. Catechol-O-methyltransferase (COMT) genotypes and working memory: associations with differing cognitive operations. Biol Psychiatry 2005;58:901-907.

13. Goldberg TE, Egan MF, Gscheidle T, Coppola R, Weickert $\mathrm{T}$, Kolachana BS, et al. Executive subprocesses in working memory: relationship to catechol-O-methyltransferase Val158Met genotype and schizophrenia. Arch Gen Psychiatry 2003;60:889-896.

14. Egan MF, Goldberg TE, Kolachana BS, Callicott JH, Mazzanti CM, Straub RE, et al. Effect of COMT Val108/158 Met genotype on frontal lobe function and risk for schizophrenia. Proc Natl Acad Sci U S A 2001;98:69176922.

15. Diamond A, Briand L, Fossella J, Gehlbach L. Genetic and neurochemical modulation of prefrontal cognitive functions in children. Am J Psychiatry 2004;161:125-132.

16. Cheon KA, Jun JY, Cho DY. Association of the catecholO-methyltransferase polymorphism with methylphenidate response in a classroom setting in children with attentiondeficit hyperactivity disorder. Int Clin Psychopharmacol 2008;23:291-298.

17. Bellgrove MA, Domschke K, Hawi Z, Kirley A, Mullins C, Robertson $\mathrm{IH}$, et al. The methionine allele of the COMT polymorphism impairs prefrontal cognition in children and adolescents with ADHD. Exp Brain Res 2005;163:352-360.

18. Kereszturi E, Tarnok Z, Bognar E, Lakatos K, Farkas L, Gadoros J, et al. Catechol-O-methyltransferase Val158Met polymorphism is associated with methylphenidate response in ADHD children. Am J Med Genet B Neuropsychiatr Genet 2008;147B:1431-1435.

19. Barr CL, Wigg K, Malone M, Schachar R, Tannock R, Roberts W, et al. Linkage study of catechol-O-methyltransferase and attention-deficit hyperactivity disorder. Am J Med Genet 1999;88:710-713.

20. Hawi Z, Millar N, Daly G, Fitzgerald M, Gill M. No association between catechol-O-methyltransferase (COMT) gene polymorphism and attention deficit hyperactivity disorder (ADHD) in an Irish sample. Am J Med Genet 2000; 96:282-284.

21. Tahir E, Curran S, Yazgan Y, Ozbay F, Cirakoglu B, Asherson PJ. No association between low- and high-activity catecholamine-methyl-transferase (COMT) and attention deficit hyperactivity disorder (ADHD) in a sample of Turkish children. Am J Med Genet 2000;96:285-288.

22. Cheuk DK, Wong V. Meta-analysis of association between a catechol-O-methyltransferase gene polymorphism and 
attention deficit hyperactivity disorder. Behav Genet 2006; 36:651-659.

23. Gizer IR, Ficks C, Waldman ID. Candidate gene studies of ADHD: a meta-analytic review. Hum Genet 2009;126:5190.

24. Courvoisie H, Hooper SR, Fine C, Kwock L, Castillo M. Neurometabolic functioning and neuropsychological correlates in children with ADHD-H: preliminary findings. $J$ Neuropsychiatry Clin Neurosci 2004;16:63-69.

25. Husarova V, Bittsansky M, Ondrejka I, Dobrota D. Prefrontal grey and white matter neurometabolite changes after atomoxetine and methylphenidate in children with attention deficit/hyperactivity disorder: a (1)H magnetic resonance spectroscopy study. Psychiatry Res 2014;222:75-83.

26. Amor LB. ${ }^{1}$ H-Magnetic resonance spectroscopy study of stimulant medication effect on brain metabolites in French Canadian children with attention deficit hyperactivity disorder. Neuropsychiatr Dis Treat 2014;10:47-54.

27. Aoki Y, Inokuchi R, Suwa H, Aoki A. Age-related change of neurochemical abnormality in attention-deficit hyperactivity disorder: a meta-analysis. Neurosci Biobehav Rev 2013;37:1692-1701.

28. American Psychiatric Association. Diagnostic and statistical manual of mental disorders. Text revision. 4th ed. Washington, DC:American Psychiatric Association;2000.

29. First MB, Spitzer RL, Gibbon M, Williams JBW. Structured clinical interview for DSM-IV clinical version (SCID-I CV). Washington, DC:American Psychiatric Press Inc.;1997. p.1-87.

30. Gunay S, Savran C, Aksoy UM, Maner F, Turgay A, Yargıç I. [The norm study, transliteral equivalence, validity, reliability of adult hyperactivity scale in Turkish adult population]. Türkiye'de Psikiyatri 2006;8:98-107. Turkish.

31. Miller SA, Dykes DD, Polesky HF. A simple salting out procedure for extracting DNA from human nucleated cells. Nucleic Acids Res 1988;16:1215.

32. Yatsuga C, Toyohisa D, Fujisawa TX, Nishitani S, Shinohara $\mathrm{K}$. Matsuura N, et al. No association between catecholO-methyltransferase (COMT) genotype and attention deficit hyperactivity disorder (ADHD) in Japanese children. Brain Dev 2014;36:620-625.

33. Carrey N, MacMaster FP, Fogel J, Sparkes S, Waschbusch $\mathrm{D}$, Sullivan $\mathrm{S}$, et al. Metabolite changes resulting from treatment in children with ADHD: a 1H-MRS study. Clin Neuropharmacol 2003;26:218-221.

34. Faraone SV, Biederman J, Mick E. The age-dependent decline of attention deficit hyperactivity disorder: a metaanalysis of follow-up studies. Psychol Med 2006;36: 159-165.

35. Soares DP, Law M. Magnetic resonance spectroscopy of the brain: review of metabolites and clinical applications. Clin Radiol 2009;64:12-21.

36. Guney E, Senol S, Sener S. [Neuroimaging methods in attention deficit hyperactivity disorder]. Klinik Psikiyatri 2008;11:84-94. Turkish.

37. Amen DG, Carmichael BD. High-resolution brain SPECT imaging in ADHD. Ann Clin Psychiatry 1997;9:81-86.

38. Kim BN, Lee JS, Cho SC, Lee DS. Methylphenidate increased regional cerebral blood flow in subjects with attention deficit/hyperactivity disorder. Yonsei Med J 2001; 42:19-29.

39. Fagundes AO, Rezin GT, Zanette F, Grandi E, Assis LC, Dal-Pizzol F, et al. Chronic administration of methylphenidate activates mitochondrial respiratory chain in brain of young rats. Int J Dev Neurosci 2007;25:47-51.
40. Czerniak SM, Sikoglu EM, King JA, Kennedy DN, Mick E, Frazier J, et al. Areas of the brain modulated by single-dose methylphenidate treatment in youth with ADHD during task-based fMRI: a systematic review. Harv Rev Psychiatry 2013;21:151-162.

41. Angelie E, Bonmartin A, Boudraa A, Gonnaud PM, Mallet JJ, Sappey-Marinier D. Regional differences and metabolic changes in normal aging of the human brain: proton MR spectroscopic imaging study. AJNR Am J Neuroradiol 2001; $22 \cdot 119-127$

42. Jin Z, Zang YF, Zeng YW, Zhang L, Wang YF. Striatal neuronal loss or dysfunction and choline rise in children with attention-deficit hyperactivity disorder: a 1H-magnetic resonance spectroscopy study. Neurosci Lett 2001;315: 45-48.

43. Fayed N, Modrego PJ, Castillo J, Dávila J. Evidence of brain dysfunction in attention deficit-hyperactivity disorder: a controlled study with proton magnetic resonance spectroscopy. Acad Radiol 2007;14:1029-1035.

44. Wiguna T, Guerrero AP, Wibisono S, Sastroasmoro S. Effect of 12-week administration of 20-mg long-acting methylphenidate on $\mathrm{Glu} / \mathrm{Cr}, \mathrm{NAA} / \mathrm{Cr}$, Cho/Cr, and $\mathrm{mI} / \mathrm{Cr}$ ratios in the prefrontal cortices of school-age children in Indonesia: a study using $1 \mathrm{H}$ magnetic resonance spectroscopy (MRS). Clin Neuropharmacol 2012;35:81-85.

45. Kato $T$, Nishina $M$, Matsushita $K$, Hori $E$, Mito $T$, Takashima S. Neuronal maturation and N-acetyl-L-aspartic acid development in human fetal and child brains. Brain Dev 1997;19:131-133.

46. Proal E, Reiss PT, Klein RG, Mannuzza S, Gotimer K, Ramos-Olazagasti MA, et al. Brain gray matter deficits at 33-year follow-up in adults with attention-deficit/hyperactivity disorder established in childhood. Arch Gen Psychiatry 2011;68:1122-1134.

47. Almeida LG, Ricardo-Garcell J, Prado H, Barajas L, Fernández-Bouzas A, Avila $\mathrm{D}$, et al. Reduced right frontal cortical thickness in children, adolescents and adults with $A D H D$ and its correlation to clinical variables: a crosssectional study. J Psychiatr Res 2010;44:1214-1223.

48. Kronenberg G, Ende G, Alm B, Deuschle M, Heuser I, Colla M. Increased NAA and reduced choline levels in the anterior cingulum following chronic methylphenidate. A spectroscopic test-retest study in adult ADHD. Eur Arch Psychiatry Clin Neurosci 2008;258:446-450.

49. Russell VA, Oades RD, Tannock R, Killeen PR, Auerbach JG, Johansen EB, et al. Response variability in AttentionDeficit/Hyperactivity Disorder: a neuronal and glial energetics hypothesis. Behav Brain Funct 2006;2:30.

50. Yang P, Wu MT, Dung SS, Ko CW. Short-TE proton magnetic resonance spectroscopy investigation in adolescents with attention-deficit hyperactivity disorder. Psychiatry Res 2010;181:199-203.

51. Bates TE, Strangward M, Keelan J, Davey GP, Munro PM, Clark JB. Inhibition of $\mathrm{N}$-acetylaspartate production: implications for ${ }^{1} H$ MRS studies in vivo. Neuroreport 1996;7: 1397-1400.

52. Carrey NJ, MacMaster FP, Gaudet L, Schmidt MH. Striatal creatine and glutamate/glutamine in attention-deficit/hyperactivity disorder. J Child Adolesc Psychopharmacol 2007; 17:11-17.

53. Nyberg F. Structural plasticity of the brain to psychostimulant use. Neuropharmacology 2014;87:115-124.

54. Dietz DM, Dietz KC, Nestler EJ, Russo SJ. Molecular mechanisms of psychostimulant-induced structural plasticity. Pharmacopsychiatry 2009;42 Suppl 1:S69-S78. 
55. Spencer TJ, Brown A, Seidman LJ, Valera EM, Makris N, Lomedico A, et al. Effect of psychostimulants on brain structure and function in ADHD: a qualitative literature review of magnetic resonance imaging-based neuroimaging studies. J Clin Psychiatry 2013;74:902-917.

56. Bledsoe J, Semrud-Clikeman M, Pliszka SR. A magnetic resonance imaging study of the cerebellar vermis in chronically treated and treatment-naïve children with attention-deficit/hyperactivity disorder combined type. Biol Psychiatry 2009;65:620-624.

57. Semrud-Clikeman M, Pliśzka SR, Lancaster J, Liotti M. Volumetric MRI differences in treatment-naïve vs chronically treated children with ADHD. Neurology 2006;67: 1023-1027.

58. Sobel LJ, Bansal R, Maia TV, Sanchez J, Mazzone L, Durkin K, et al. Basal ganglia surface morphology and the effects of stimulant medications in youth with attention deficit hyperactivity disorder. Am J Psychiatry 2010;167: 977-986.

59. Shaw P, Sharp WS, Morrison M, Eckstrand K, Greenstein DK, Clasen LS, et al. Psychostimulant treatment and the developing cortex in attention deficit hyperactivity disorder. Am J Psychiatry 2009; 166:58-63.

60. Castellanos FX, Lee PP, Sharp W, Jeffries NO, Greenstein DK, Clasen LS, et al. Developmental trajectories of brain volume abnormalities in children and adolescents with attention-deficit/hyperactivity disorder. JAMA 2002;288: 1740-1748.

61. Schmidt S, Petermann F. Developmental psychopathology: Attention deficit hyperactivity disorder (ADHD). BMC Psychiatry 2009;9:58. 